\title{
Advancements in Decontamination of Vacuum Systems Using Plasma Cleaning
}

\author{
Ronald Vane ${ }^{1}$ and C. A. Moore ${ }^{1}$ \\ 1. XEI Scientific, Redwood City, CA, USA
}

In general vacuum-based processes can suffer deleterious effects from the presence of adventitious hydrocarbons that result from various sources such as oils and solvents as well as work-pieces. For example in electron microscopy the presence of hydrocarbons gives rise to unwelcome effects such as image blur as well 'black square' formation during lengthy beam exposure times [1]. These and related issues have given rise to use of RF-driven plasmas to decontaminate vacuum chambers via generation of excited-state species (typically oxygen) that gently remove contamination. It is important to note that these systems generate electrically neutral cleaning species that flow from the plasma into the chamber so the decontamination is removed by chemical reactions.

Last year at this conference XEI Scientific introduced the Evactron ${ }^{\circledR}$ Zephyr which is a result of our efforts to better understand the science of plasma cleaning and subsequently develop new generation tools with a number of improvements including the ability to clean at pressures compatible with operating turbo-molecular pumps (TMPs). This latter requirement presents a difficulty as the low pressures favourable to TMPs are not conducive to plasma ignition- starting a plasma is generally easier in the 0.5 to 5 Torr range (i.e., at the minimum of the Paschen curve). The Evactron ${ }^{\circledR}$ Zephyr represents a method to resolve these competing pressure requirements.

The operation of the revised tool can be understood by examining the measured chamber vacuum during the cleaning cycle. The Evactron cleaning interlock requires that the electron gun be closed and high voltages be off before the cleaning begins, and this insured detecting a downward pressure change at 2 Torr. In Figure 1a classic Evactron cleaning begins by sensing that the pressure has decreased to $<2$ Torr and then controlling the ignition pressure to selected value. Upon ignition the pressure then controls to another value to clean for a controlled duration; this system had a lower limit of about $150 \mathrm{~m}$ Torr. In the Zephyr model, new valves and electrode changes allow operation of the cleaning plasma at pressures between 10 and 25 mTorr depending on pumping speed. These pressures are compatible with full speed TMP operation with flow rates of between 10-25 sccm. (Lower flows and pressures starve the cleaning process of reactive species and slow down cleaning.) These features are evident in Figure 1b where ignition occurs at 250 mTorr and plasma cleaning now occurs at 20 mTorr.

In order to quantitatively compare the efficacy of the plasma cleaning we have developed a metrology technique that has been shown to be both stable and repeatable for durations $>20$ hours [2]. Briefly we use a commercial quartz crystal microbalance (QCM) wherein the crystal is coated with a hydrocarbon using a proprietary process. By locating the crystal and holder in the vacuum chamber we have performed detailed studies of how changes in conditions (both parametric variations as well as system design modifications) affect the plasma cleaning process.

Using this method we have obtained the results of Figure 2 that shows the increase in cleaning rate as the pressure is reduced. These data clearly demonstrate the need for the Evactron Zephyr as it allows ignition of the plasma at higher pressure followed by the reduction of pressure to the higher cleaning rate regime. For sake of comparison we also replaced the capacitively coupled Zephyr plasma system and installed a inductively coupled plasma (ICP) system. All other factors including power delivered to 
the plasma were held constant. The pressure values used in Figure 2 was measured on an independent gauge and is indicative of the chamber pressure. From these data it is clear that the Zephyr system strongly outperforms the ICP system over the ranges of pressures optimal for plasma decontamination.

References:

[1] András E. Vladár, Michael T. Postek and Ronald Vane, "Active Monitoring and Control of Electron Beam Induced Contamination”, Proc. SPIE Vol. 4344 (2001), 835.

[2] E. Kosmowska, D. Varley, R. Vane, and C. Moore, SPIE Conference on Lithography (2013).

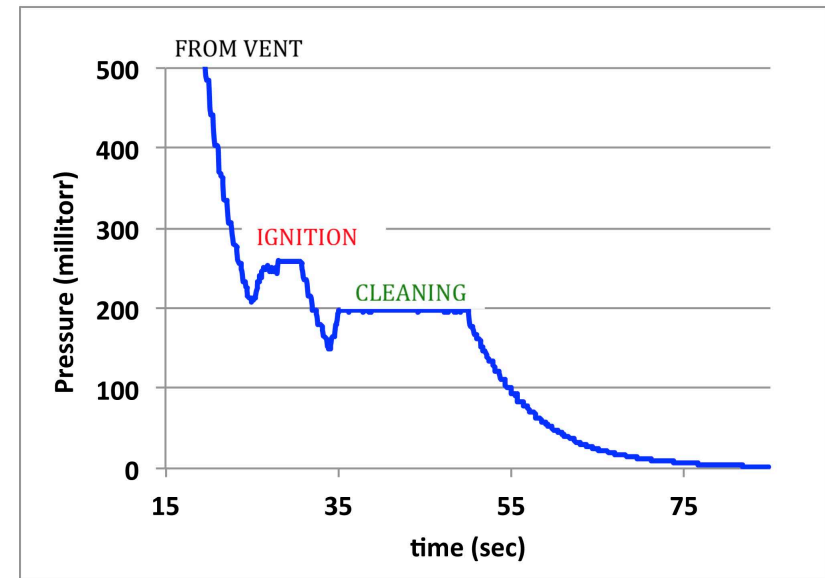

1a. Classic Evactron

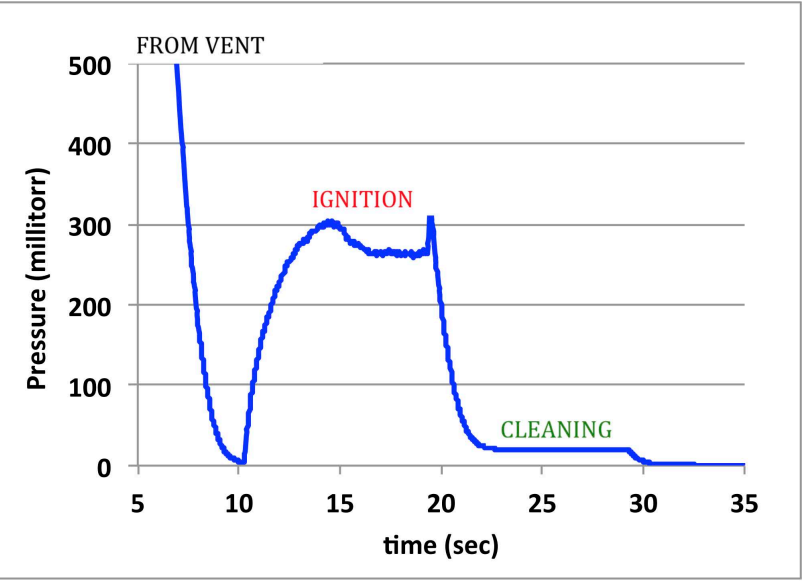

1b. Evactron Zephyr

Figure 1. Temporal measurement of chamber pressure during cleaning cycle, comparing classic Evactron and Evactron Zephyr. Note change in horizontal axis scale.

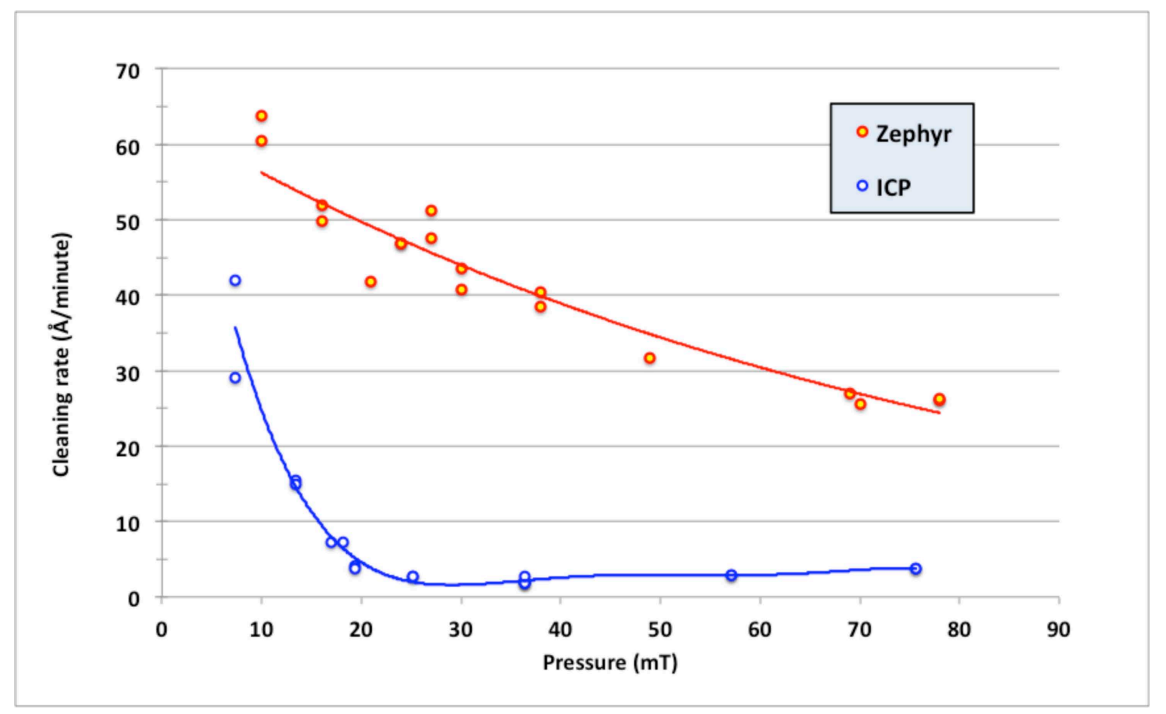

Figure 2. Comparison of cleaning (hydrocarbon removal) rate versus pressure obtained XEI Evactron Zephyr and ICP-based plasma (delivered power of 20 Watts for each). 